\title{
Fermi Surface Topology and Low-Lying Quasiparticle Dynamics of Parent $\mathrm{Fe}_{1+x} \mathrm{Te} / \mathrm{Se}$ Superconductor
}

\author{
Y. Xia, ${ }^{1}$ D. Qian, ${ }^{1,2}$ L. Wray, ${ }^{1,3}$ D. Hsieh, ${ }^{1}$ G. F. Chen, ${ }^{4}$ J. L. Luo, ${ }^{4}$ N. L. Wang, ${ }^{4}$ and M. Z. Hasan ${ }^{1,5, *}$ \\ ${ }^{1}$ Joseph Henry Laboratories of Physics, Department of Physics, Princeton University, Princeton, New Jersey 08544, USA \\ ${ }^{2}$ Department of Physics, Shanghai Jiao Tong University, Shanghai 200030, China \\ ${ }^{3}$ Lawrence Berkeley National Laboratory, University of California, Berkeley, California 94305, USA \\ ${ }^{4}$ Beijing National Laboratory for Condensed Matter Physics, Institute of Physics, Chinese Academy of Sciences, Beijing, China \\ ${ }^{5}$ Princeton Center for Complex Materials, Princeton University, Princeton, New Jersey 08544, USA
}

(Received 16 January 2009; published 13 July 2009)

\begin{abstract}
We report the first photoemission study of $\mathrm{Fe}_{1+x} \mathrm{Te}$ - the host compound of the newly discovered ironchalcogenide superconductors (maximum $T_{c} \sim 27 \mathrm{~K}$ ). Our results reveal a pair of nearly electron-hole compensated Fermi pockets, strong Fermi velocity renormalization, and an absence of a spin-densitywave gap. A shadow hole pocket is observed at the " $X$ " point of the Brillouin zone which is consistent with a long-range ordered magnetostructural ground state. No signature of Fermi surface nesting instability associated with $\mathbf{Q}=(\pi / 2, \pi / 2)$ is observed. Our results collectively reveal that the $\mathrm{Fe}_{1+x}$ Te series is different from the undoped phases of the high $T_{c}$ pnictides and likely harbor an unusual mechanism for superconductivity and magnetic order.
\end{abstract}

DOI: 10.1103/PhysRevLett.103.037002

PACS numbers: 74.70. $-\mathrm{b}, 74.25 . J b, 79.60 .-\mathrm{i}$

The discovery of superconductivity in the pnictides (FeAs-based compounds) has generated interest in understanding the general interplay of quantum magnetism, electronic structure, and superconductivity in iron-based layered compounds [1,2]. The very recent observation of unusual superconductivity and magnetic order in the structurally simpler compounds such as $\mathrm{FeSe}$ and $\mathrm{Fe}_{1+x} \mathrm{Te}$ is the highlight of current iron-based high $T_{c}$ research [3-7]. The expectation is that these compounds may provide a way to isolate the key ingredients for superconductivity and the nature of the parent magnetically order state which may potentially differentiate between vastly different theoretical models [8-10] due to their significantly simpler crystal structure. Superconductivity with transition temperature up to $15 \mathrm{~K}$ is achieved in the $\mathrm{Fe}_{1+x}(\mathrm{Se}, \mathrm{Te})$ series [3,4] and $T_{c}$ increases up to $27 \mathrm{~K}$ under a modest application of pressure [5]. Density functional theories (DFT) predict that the electronic structure is very similar to the iron pnictides and magnetic order in FeTe originates from very strong Fermi surface (FS) nesting leading to the largest spindensity wave (SDW) gap in the series. Consequently, the doped FeTe compounds are expected to exhibit $T_{c}$ much higher than that observed in FeSe if superconductivity would indeed be originating from the so-called $(\pi, 0)$ spin fluctuations [10]. These predictions critically base their origin on the Fermi surface topology and the lowlying quasiparticle structure; however, no experimental results on the Fermi model exist on this sample class to this date. Here we report the first angle-resolved photoemission spectroscopy (ARPES) study of the $\mathrm{Fe}_{1+x} \mathrm{Te}-$ the host compound of the superconductor series. Our results reveal a pair of nearly electron-hole compensated Fermi pockets, significant band renormalization, and a remarkable absence of the large SDW gap. Although the observed Fermi surface topology is broadly consistent with the DFT calculations, no Fermi surface nesting instability associated with the magnetic ordering wave vector was observed. Our measurements reported here collectively suggest that the FeTe compound series is different from the parent compound of the pnictide superconductors and may harbor novel forms of magnetic and superconducting instabilities not present in the high $T_{c}$ pnictides.

Single crystals of $\mathrm{Fe}_{1+x} \mathrm{Te}$ were grown using the Bridgeman technique. A mixture of ground $\mathrm{Fe}$ and $\mathrm{Te}$ powder was heated to $920^{\circ} \mathrm{C}$ in an evacuated tube then slowly cooled, forming single crystals. The iron concentration was measured by inductively coupled plasma technique and $x$ was determined to be less than 0.05 . The crystal structure of these superconductors is comprised of a direct stacking of tetrahedral FeTe layers along the $c$ axis bonded by weak van der Waals coupling along which the samples are easily cleaved. High-resolution ARPES measurements were then performed using linearly polarized $40 \mathrm{eV}$ photons on beam line 10.0.1 of the Advanced Light Source at the Lawrence Berkeley National Laboratory. The energy and momentum resolution was $15 \mathrm{meV}$ and $2 \%$ of the Brillouin zone (BZ) using a Scienta analyzer. The in-plane crystal orientation was determined by Laue $\mathrm{x}$-ray diffraction prior to inserting into the ultrahigh vacuum measurement chamber. The magnetic order below $65 \mathrm{~K}$ was confirmed by dc susceptibility measurements (Fig. 1). The samples were cleaved in situ at $10 \mathrm{~K}$ under pressures of less than $5 \times 10^{-11}$ torr, resulting in shiny flat surfaces. Cleavage properties were characterized by STM topography and by examining the optical reflection properties.

Figure 2 presents the momentum dependence of the photoemission intensity $n(k)$ at $10 \mathrm{~K}$ at various binding 


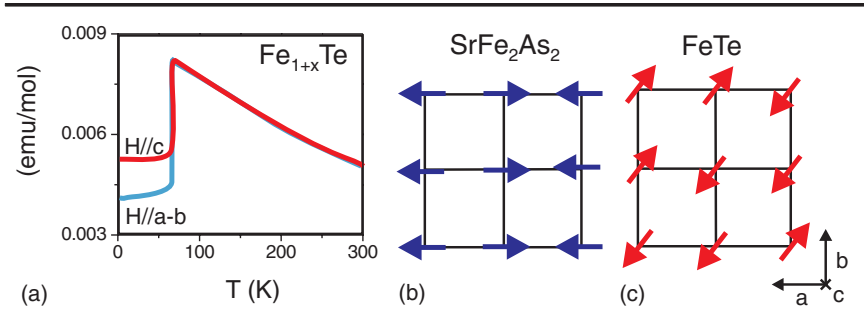

FIG. 1 (color online). Magnetostructural transition and longrange order in $\mathrm{Fe}_{1+x} \mathrm{Te}$. (a) Temperature dependence of the magnetic susceptibility [6]. (b) The spins in $\mathrm{SrFe}_{2} \mathrm{As}_{2}$ are collinear and $\mathbf{Q}_{\mathrm{AFM}}$ points along the $(\pi, 0)$ direction. (c) The ordering vector, $\mathbf{Q}_{\mathrm{AFM}}$ in $\mathrm{FeTe}$, is rotated by $45^{\circ}$ and points along the $\left(\frac{\pi}{2}, \frac{\pi}{2}\right)$ direction [15].

energies $(0,-20,-40$, and $-60 \mathrm{meV})$, integrated over an energy window of $\pm 5 \mathrm{meV}$ at each binding energy. The nonzero spectral intensity at the Fermi level $(\mu)$ confirmed that the low temperature state of $\mathrm{Fe}_{1+x} \mathrm{Te}$ is metalliclike. At the Fermi level, electrons are mostly distributed in one broad holelike pocket at $\Gamma$ and another similar size electronlike pocket around $M$. To reveal the band shapes we present a gradual binding-energy evolution of the band features and present the data in a way to simulate the effect of rigidly lowering of the chemical potential down to $60 \mathrm{meV}$. The FS (at $0 \mathrm{meV}$ ) is seen to consist of circular hole pockets centered at $\Gamma$, and elliptical electron pockets at the $M$ points. An elliptical-like feature at the $M$ point expands as the binding energy is increased, suggesting that the associated band is holelike. We will subsequently show that this band lies below $\mu$ and the $M$-point Fermi pockets are only electronlike [2(e)]. By considering the dominant intensity patterns in Figs. 2(a)-2(d), apart from a weak

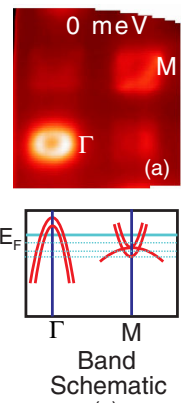

(e)
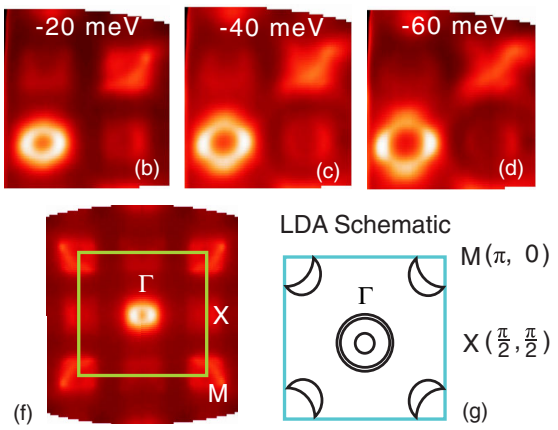

FIG. 2 (color online). Fermi surface topology. (a) Fermi surface topology of FeTe. (a)-(d) Connection to the underlying band structure is revealed by considering the evolution of the density of states $n(k)$ as the chemical potential is rigidly lowered by 20,40 , and $60 \mathrm{meV}$. The Fermi surface consists of hole pockets centered at $\Gamma$ and electron pockets centered at $M$. An additional holelike feature is observed at $M$ in (b)-(d), which is attributed to a band lying below $E_{F}$ [see schematic in (e)]. (e) presents a schematic of dispersions in the $\Gamma-M$ direction forming the electron and hole pockets. A qualitative agreement is observed between (f) the experimentally measured FS topology and (g) the local-density approximation (LDA) calculated [10] FS although measured bands are renormalized. feature at " $X$ " point, the FS topology is similar to that expected from the DFT calculations $[2(\mathrm{~g})][10]$. In addition, the area of the holelike FS pocket at $\Gamma$ and the electronlike FS pocket at $M$ are approximately equal in size suggesting nearly equal number of electron and hole carrier densities in this material. Therefore, if the excess Fe atoms are contributing to the carrier density it is likely to be small and beyond our $k$ resolution of the experiment.

In order to systematically study the low-lying energy band structure, ARPES spectra are taken along different $k$ space cut directions in the 2D BZ. Two different electronphoton scattering geometries are used to ensure that all bands are imaged. In a $\sigma$ scattering geometry, where the polarization vector is along $k_{y}$ and the detector slit is along $k_{x}$, photoelectron signal is predominantly from the $d_{x y}$ and $d_{y z}$ energy bands due to the dipole emission matrix elements $[11,12]$. Similarly, when the detector slit is along $k_{y}$, the $\pi$ geometry, the $d_{x z}, d_{z^{2}}$, and $d_{x^{2}-y^{2}}$ states are predominantly excited. Figures 3(a) and 3(b) present scans along the $\Gamma-M$ direction in the $\sigma$ and $\pi$ geometries. In both sets of spectra, one finds a broad holelike band centered at $\Gamma$. However, near $M$ the scans taken at two different geometries are drastically different. In cut 1 , two holelike bands $\left[\alpha_{2}, \alpha_{3}\right.$ as marked in 3(a)] are observed to be approaching $\mu$. Under the $\pi$ geometry the band emission pattern near $M$ is dramatically different (see the $\beta_{1}$ band in cut 2), while the $\alpha_{2}$ and $\alpha_{3}$ band signals are significantly weaker. The polarization dependence suggests that the $\beta_{1}$ band should have $d_{x z}, d_{z^{2}}$, or $d_{x^{2}-y^{2}}$ orbital character.

In order to fully resolve the broad band feature centered at $\Gamma$, high-resolution scans are performed along different $k$-cut directions through the zone center. Figure 3(c) presents one cut in the $\Gamma-M$ direction inside the first zone, together with the corresponding energy distribution curve (EDC). Two holelike bands are resolved, labeled $\alpha_{2}$ and $\alpha_{3}$. Since there are traces of multiple bands near $M$, one might wonder whether the $\alpha_{2}$ band crosses $\mu$ near the $M$ point, thus forming a holelike Fermi pocket. To systematically investigate this, a series of spectra are taken along the $\Gamma-M$ direction with $\pi$ geometry [3(d)-3(f)] through multiple $k$ cuts intersecting the $M$-point Fermi pocket. At $M$, cut 4 shows a strong electronlike band forming the FS pocket. This band can be attributed to the $\beta_{1}$ band (also observed in cut 2). As one moves away from $M$, the band intensity becomes increasingly more holelike, indicating the emergence of the $\alpha_{2}$ band. Nevertheless, the hole band lies completely and consistently below the Fermi level, with some weak electron quasiparticle intensity above the band maximum. The $\beta_{1}$ intensity becomes the weakest near the edge of the $M$ pocket (cut 6). But even at that location, the hole pocket lies at least $10 \mathrm{meV}$ below the chemical potential. The result shows that there are no hole pocket features in the FS near $M$, which supports the interpretation that this material is nearly electron-hole compensated. Additionally, one can map the observed bands to the band structure estimated by DFT calculations 

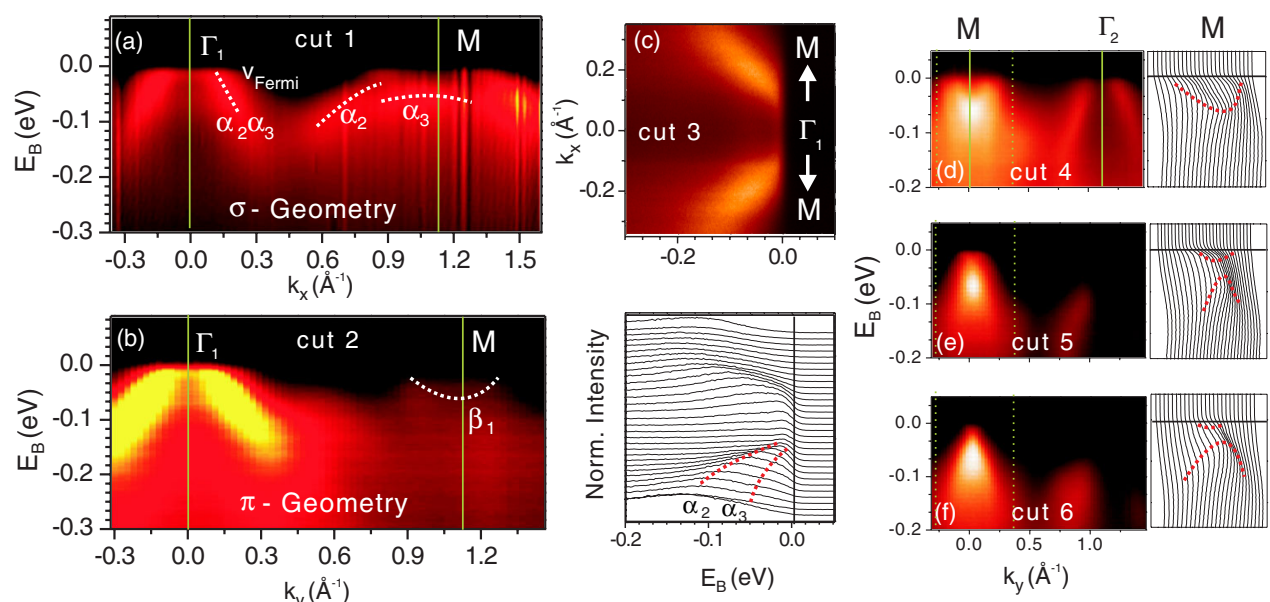

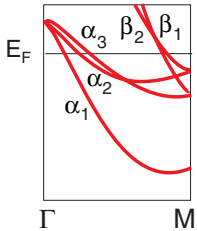

(g)

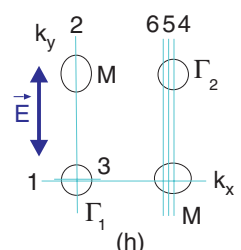

(h)

FIG. 3 (color online). Polarization-resolved study reveals the low-lying band topology. ARPES spectra along the $\Gamma-M$ direction in the (a) $\sigma$-and (b) $\pi$-scattering geometries. $v_{F}$ of the quasiparticle band forming the $\Gamma$ hole pocket is calculated from the dashed line. (c) The EDC of a high momentum resolution scan through $\Gamma$ shows two holelike bands crossing the $E_{F}$. (d)-(f) Cuts along the $M$ - $\Gamma$ direction through the electron FS pocket show that the hole band near $M$ remains at least $10 \mathrm{meV}$ below $E_{F}$. For each scan direction the corresponding EDC is also presented, within the momentum range marked by green dashed lines. Comparison with (g) a band dispersion schematic of the LDA result [10]. (h) The directions of the six scans are identified in the first BZ.

[schematic in $3(\mathrm{~g})$ [10]]. The calculation finds three bands $\left(\alpha_{1}-\alpha_{3}\right)$ crossing Fermi level near $\Gamma$ and two $\left(\beta_{1}, \beta_{2}\right)$ near $M$, forming the electron and hole pockets. While the DFT calculated bands agree fairly well with our data, within our resolution or the sample surface roughness, we have not succeeded in fully resolving the $\alpha_{1}$ band near $\Gamma$. The $\beta_{2}$ band, which forms the second $M$ electron pocket, is observed in the Fermi surface topology (Fig. 2). The overall band narrowing is about factor of 2 compared to the DFT calculations highlighting the importance of correlation effects. This is also consistent with a small Fermi velocity $\left(v_{\text {Fermi }} \sim 0.7 \mathrm{eV} \cdot \AA\right)$ observed [Fig. 3(a)].

We now revisit the details of the FS map (Fig. 2) and discuss a weak feature observed at $X=\left(\frac{\pi}{2}, \frac{\pi}{2}\right)$ between two $\Gamma$ points [Fig. 4(e)]. Such a feature is not expected from the DFT calculations. A similar feature, whose origin is debated, has been observed in $A \mathrm{Fe}_{2} \mathrm{As}_{2}(A=\mathrm{Ba}, \mathrm{Sr})$, which is attributed to a $2 \times 1$ surface reconstruction of the $A$ atom layer $[13,14]$, in addition to a weak bulk structural distortion. However, in FeTe there are no additional atoms (such as $\mathrm{Sr}$ or $\mathrm{Ba}$ ) between the $\mathrm{Fe}$ layers and the crystal cleaves at a weak bond between two adjacent layers. Therefore, no strong $2 \times 1$ long-range ordered $A$-type reconstruction is expected except for a weak bulklike structural orthorhombicity tied to the magnetic order (magnetostructural effect). Recent neutron $[15,16]$ and x-ray diffraction $[17,18]$ studies have shown that FeTe undergoes a bulk structural distortion from the tetragonal to weakly monoclinic or orthorhombic phase near $65 \mathrm{~K}$, accompanied by long-range magnetic order $\mathbf{Q}_{\mathrm{AFM}}=\left(\frac{\pi}{2}, \frac{\pi}{2}\right)$.

In the parent compound of the pnictide superconductors such as the $\mathrm{SrFe}_{2} \mathrm{As}_{2}$ or $\mathrm{BaFe}_{2} \mathrm{As}_{2}$, the SDW vector $\mathbf{Q}_{\mathrm{SDW}}=(\pi, 0)$ coincides with a Fermi surface nesting vector connecting the hole pocket at $\Gamma$ and the electron pocket at $M$. Currently, it is believed that this nesting is responsible for opening a gap in the low temperature physical properties $[6,11,19]$. In the case of $\mathrm{FeTe}$, while a nesting vector can indeed be drawn along $\mathbf{Q}_{\mathrm{SDW}}=(\pi, 0)$ between a pair of electron and hole pockets, all available neutron scattering measurements report that the antiferromagnetic ordering vector is $45^{\circ}$ away from that in $\mathrm{SrFe}_{2} \mathrm{As}_{2}$, namely, in $\mathrm{Fe}_{1+x} \mathrm{Te}, \mathbf{Q}_{\mathrm{AFM}}=\left(\frac{\pi}{2}, \frac{\pi}{2}\right)$. The ordering shows a commensurate to incommensurate crossover if the concentration of excess iron $x$ is increased. Another remarkable difference is that the magnetic susceptibility is Curie-Weiss-like in FeTe suggesting that the magnetism is of local-moment origin. Within a local-moment-like AFM long-range ordered state which also couples to a weak structural distortion, one should expect relatively intense shadow Fermi surfaces along the Néel vector $\mathbf{Q}_{\mathrm{AFM}}$. The $X$-point Fermi surface we observe thus can be related to the vector observed in neutron scattering $X=\Gamma+\mathbf{Q}_{\mathrm{AFM}}$. The weak shadowlike $X$-pocket FS might therefore arise from a band folding due to long-range magnetic order. We also caution that a slight surface distortion is another possible interpretation of the data. However, unlike $\mathrm{SrFe}_{2} \mathrm{Se}_{2}$, where the $\Gamma$ electron pocket nests with the $M$ hole pockets via $\mathbf{Q}_{\mathrm{SDW}}=(\pi, 0)$ [20], an analogous nesting channel is unavailable at $\left(\frac{\pi}{2}, \frac{\pi}{2}\right)$ in the FeTe [4(b)], clearly ruling out FS nesting as the origin of magnetic order. In the absence of nesting FS gapping is not expected in FeTe, which is consistent with our results in Figs. 2 and 3. A large low temperature specific heat value [6] is thus consistent with our observation of a Fermi surface in the correlated magnetostructurally ordered state. To examine the band dispersion character of the $X$ pocket, Fig. 4(f) presents a spectra along the $\Gamma-X$ direction. Results show two bands dispersing towards $\mu$, forming the hole pocket in the " $X$ " FS 

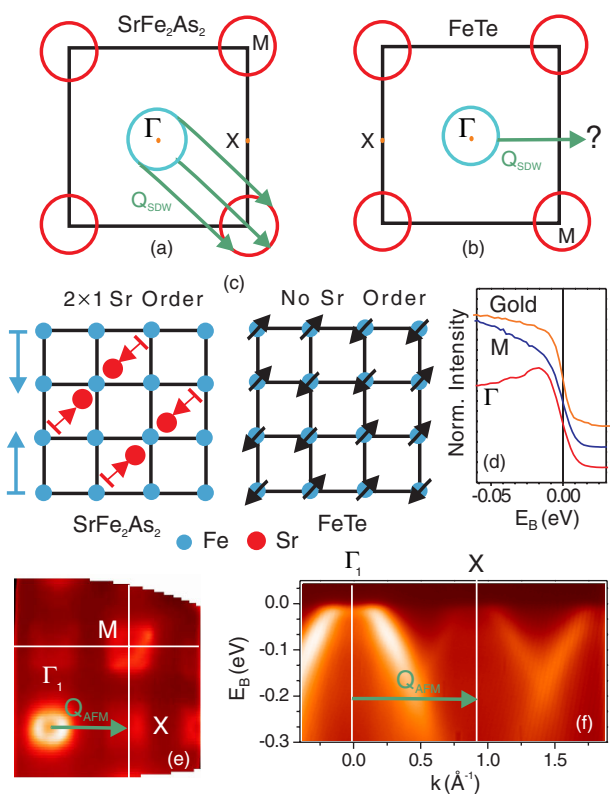

FIG. 4 (color online). Electronic structure and Magnetic ordering mechanism. While (a) the electron and hole pockets in $\mathrm{SrFe}_{2} \mathrm{As}_{2}$ can be kinematically nested by $\mathbf{Q}_{\mathrm{AFM}}=(\pi, 0)$, there exists (b) no FS pocket which can be nested by $\mathbf{Q}_{\mathrm{AFM}}=\left(\frac{\pi}{2}, \frac{\pi}{2}\right)$ in the FeTe BZ. A FS pocket observed at $X$ in $\mathrm{SrFe}_{2} \mathrm{As}_{2}$ has been attributed to the (c) $2 \times 1$ surface order of the $\mathrm{Sr}$ atomic layer (red), which occurs in addition to a weak bulk distortion (blue) across the SDW transition. However, there are no additional $\mathrm{Sr}$ atoms between the Fe layers in FeTe, so no $\mathrm{Sr}$ order is possible, although a weak bulk or surface distortion is not excluded. (d) The EDCs measured at near the $\Gamma$ and $M$ pockets exhibit no evidence of energy gaps. Nevertheless, (e) a weak FS pocket is observed at $X$, (f) corresponding to two holelike bands dispersing towards the chemical potential.

whose shapes are indeed very similar to the bands that form the central $\Gamma$-pocket FS. Further evidence against nesting comes from the absence of a large gap at the overall low temperature electron distributions presented in Figs. 2, 3, and 4(d). Our results suggest that the ground state is a nearly electron-hole compensated semimetal. Absence of a gap is consistent with bulk optical conductivity, specific heat, and Hall measurements in FeTe [6] (while most measurements do report a gap in $\mathrm{SrFe}_{2} \mathrm{As}_{2}$ [19]).

A recent density functional calculation [21] suggests that the excess $\mathrm{Fe}$ is in a valence state near $\mathrm{Fe}^{+}$and therefore donates electron to the system. Because of the interaction of the magnetic moment of excess $\mathrm{Fe}$ with the itinerant electrons of FeTe layer, a complex magnetic ordering pattern is realized. In this scenario, excess Fe would lead to an enlargement of the electron pocket FS; however, within our resolution electron and hole Fermi surface pockets are measured to be very similar in size, suggesting a lack of substantial electron doping due to excess Fe. Finally, we note that a gapless yet long-range ordered local-moment magnetostructural ground state consistent with ARPES and neutron data taken together is captured in both first-principles electronic structure [8] and many-body spin model [9] calculations. However, the broad agreement of DFT calculations with experimental band-structure data except for about a factor 2 renormalization is also remarkable. A complete understanding of electron correlation and the true nature of the gapless state would require further study.

In conclusion, we have presented the first ARPES study of Fermi surface and polarization-resolved band topology of $\mathrm{Fe}_{1+x}$ Te. Results reveal a pair of nearly electron-hole compensated Fermi pockets, significant renormalization, and the absence of a spin-density-wave gap within our resolution. The observed new hole pocket is consistent with a long-range ordered magnetostructural ground state whereas, most remarkably, no Fermi surface nesting instability associated with the antiferromagnetic order was observed. Results collectively suggest that the $\mathrm{Fe}_{1+x} \mathrm{Te}$ series is different from the pnictides and likely harbor a novel mechanism for superconductivity and magnetism.

We thank P. W. Anderson, B. A. Bernevig, D. A. Huse, D.-H. Lee, Y. Ran, Z. Tesanovic, A. Vishwanath, and C. Xu for discussions. The synchrotron $\mathrm{X}$-ray experiments at ALS/LBNL are supported by the U.S. DOE-BES (Contract No. DE-FG02-05ER46200) and materials growth supported by NSFC and CAS China.

*To whom correspondence should be addressed. mzhasan@Princeton.edu

[1] Y. Kamihara, T. Watanabe, M. Hirano, and H. Hosono, J. Am. Chem. Soc. 130, 3296 (2008); Z. A. Ren et al., Chin. Phys. Lett. 25, 2215 (2008).

[2] G. F. Chen et al., Phys. Rev. Lett. 100, 247002 (2008); X. H. Chen et al., Nature (London) 453, 761 (2008).

[3] F. C. Hsu et al., Proc. Natl. Acad. Sci. U.S.A. 105, 14262 (2008).

[4] M. H. Fang et al., Phys. Rev. B 78, 224503 (2008).

[5] Y. Mizuguchi et al., Appl. Phys. Lett. 93, 152505 (2008).

[6] G. F. Chen et al., Phys. Rev. B 79, 140509(R) (2009).

[7] C. Xu and S. Sachdev, Nature Phys. 4, 898 (2008).

[8] F. J. Ma et al., Phys. Rev. Lett. 102, 177003 (2009).

[9] C. Fang et al., arXiv:0811.1294.

[10] A. Subedi et al., Phys. Rev. B 78, 134514 (2008).

[11] D. Hsieh et al., arXiv:0812.2289.

[12] D. Qian et al., Phys. Rev. Lett. 97, 186405 (2006).

[13] M. C. Boyer et al., arXiv:0806.4400.

[14] Y. Yin et al., Phys. Rev. Lett. 102, 097002 (2009).

[15] S. Li et al., Phys. Rev. B 79, 054503 (2009).

[16] W. Bao et al., arXiv:0809.2058.

[17] K. Yeh et al., arXiv:0808.0474.

[18] Y. Mizuguchi et al., arXiv:0810.5191.

[19] W.Z. Hu et al., Phys. Rev. Lett. 101, 257005 (2008).

[20] J. Zhao et al., Phys. Rev. B 78, 140504 (2008).

[21] L. Zhang et al., Phys. Rev. B 79, 012506 (2009). 